\title{
A Smooth System of Equations Approach to Complementarity Problems for Frictionless Contacts
}

\author{
Qin Deng, ChunGuang Li, and Hua Tang \\ State Key Laboratory of Geomechanics and Geotechnical Engineering, Institute of Rock and Soil Mechanics, \\ Chinese Academy of Sciences, Wuhan 430071, China \\ Correspondence should be addressed to Qin Deng; dengqin_x@163.com
}

Received 3 July 2015; Revised 28 August 2015; Accepted 7 September 2015

Academic Editor: Luciano Mescia

Copyright (C) 2015 Qin Deng et al. This is an open access article distributed under the Creative Commons Attribution License, which permits unrestricted use, distribution, and reproduction in any medium, provided the original work is properly cited.

Frictionless contact problems are the simplest and classical contact problems, and the contact conditions of sticking, slipping, and separation mode all can be ascribed to complementary problems. Consequently, a smooth system of equations approach for the design and analysis of complementarity problems for frictionless contacts is presented. A compute program based on boundary element technique is given and applied to two practical contact examples. The validity and accuracy of the proposed method are demonstrated.

\section{Introduction}

Contact problems are of particular importance in many engineering applications $[1,2]$ such as gears, piles, retaining walls, and tunnel lining. Since the hertz theory was developed in 1881, much research has been developed in this area including both theoretical and experimental work [3-7]. Hence it establishes the foundation for modern contact mechanics. In the analysis of contact problems, special attention must be required because the actual contact area between the contacting bodies is usually not known in advance, and the character of interface between contact bodies largely determines the deformation, movement, and stress distribution.

There is a large literature on numerical methods for contact problems. Roughly speaking, numerical algorithms can be classified into three categories. The first class is known as direct iterative algorithm [8-11], which assumes the contact region and contact status firstly and then solves the problem and checks whether the assumption is correct or not. It solves the problem iteratively by trial-and-error, thus requiring much computational effort; the second class is contact constraint algorithm [12-14], which deals with the contact boundary properly, and transforms the constrained optimization problem into nonconstrained optimization problem. According to the different methods for unconstrained optimization, the penalty method [12], Lagrange method [13], and augmented Lagrange method [14] have been introduced. The calculation accuracy based on penalty method strongly depends on the penalty coefficient, and the coefficient is decided by experience; the number of unknown variables, computation time, and memory requirement using Lagrange method are troublesome; to foster strengths and circumvent weaknesses, the augmented Lagrange method is proposed for contact problems, which decreases ill-conditioning of governing equations, and satisfies exactly constraints with finite penalties [15]. However, these methods can be generalized into the iterative algorithms. The third class is known as the mathematical programming method [16, 17], and the solutions are obtained by using either linear programming or quadratic programming techniques [18-20]. The advantage is that the original problem can be converted to programming problem by expressing the normal and tangential conditions into the complementary expression, and mature mathematical methods can be applied. Furthermore, it is known that frictionless/frictional contact problems can be formulated as complementarity problems. Hence, a numerical method for complementarity problems can be applicable to contact problems [21]. Here, the system of nonlinear equations method [22] has been widely used, which transforms the complementarity problem into a system of nonlinear equations by employing the nonlinear complementary functions. 
For numerical discretization, the boundary element method (BEM) [23] is particularly well suited to solve contact problems [24]. Except for the reduced dimensionality by one, the most striking feature of the BEM is that the tractions and displacements are computed to the same degree of accuracy, which is an important feature if reliable solutions are to be obtained [25]. The BEM was first applied to contact problems by Andersson et al. [26] in two-dimensional frictionless problems and later extended the applications to frictional problems [27]. Afterwards, other contact problems have been studied such as elastoplastic contact problems [28] and 3D frictional problems [29]. However, these problems had been solved by the iterative algorithms, and the trial-and-error, calculation accuracy and computation time, and so forth had frequently appeared. So far, the system of nonlinear equations method has not been used to solve contact problems by BEM.

In this paper, the smooth system of equations is employed to solve the two-dimensional elastic frictionless contact problems. It is comprised of the nonlinear complementary functions describing the relationship between the gap and contact pressure for any node pair and boundary integral equations. The presented approach does not need repeatedly trial calculation to decide the contact mode for any node pair. Meanwhile, the proposed method is easy to be accepted and used. According to the results of given load, the contact state can be observed obviously, and it does not need your judgment. This algorithm is implemented in a 2D BEM code and verified using two numerical examples. The results by the proposed algorithm match well with the analytical solutions and the FEM results and clearly demonstrate the feasibility and flexibility of the proposed approach for $2 \mathrm{D}$ contact analysis.

\section{Contact Problems and Its Complementarity Description}

2.1. General Description of Contact Problems. In this section, two contact bodies $\Omega^{A}$ and $\Omega^{B}$ are considered as shown in Figure 1. The boundary of any body is composed of three disjoint parts: displacement boundary $\Gamma_{u}$, traction boundary $\Gamma_{f}$, and potential contact region $\Gamma_{c}$. Nevertheless, the region $\Gamma_{c}$ has been taken sufficiently large to contain actual contact regions.

Considering a pair of points $a$ and $b$ on the bodies $\Omega^{A}$ and $\Omega^{B}$, respectively, on the contact boundary $\Gamma_{c}$, the following contact modes shown in Figure 2 may happen: separation, stick and slip mode. For the convenient description of contact conditions, it is necessary to define a local coordinate system for the pair of points $a$ and $b$. If body $\Omega^{B}$ is selected as the target body, the coordinate system should be established in point $b$ as show in Figure 3, and $n$ and $t$ denote unit normal and tangential directions, respectively. Selecting $t_{i}^{j}$ (the subscript refers to direction of force, and superscript refers to the contact point) and $g^{a b}$ (the subscript refers to the pair of points) to represent the traction and gap between the point pair, respectively, the contact conditions are listed as follows.

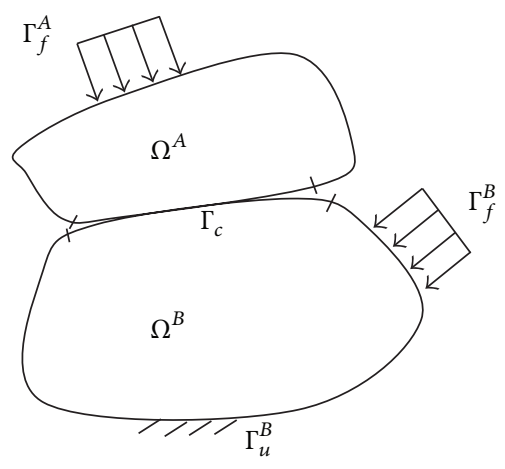

FIGURE 1: Contact of two bodies.

(1) Separation Mode. The individual traction at the pair of points $a$ and $b$ is zero, and the gap could be positive:

$$
\begin{aligned}
& t_{t}^{a}=t_{t}^{b}=0, \\
& t_{n}^{a}=t_{n}^{b}=0, \\
& g^{a b}>0 .
\end{aligned}
$$

To maintain the consistency below, the relationship $t_{n}^{a}=$ $t_{n}^{b}=0$ can be recast to $t_{n}^{a}+t_{n}^{b}=0$.

(2) Stick Mode. The individual tangential traction at the pair of points $a$ and $b$ is zero, and the total normal tractions are equal to zero. The gap should be zero:

$$
\begin{aligned}
t_{t}^{a} & =t_{t}^{b}=0, \\
t_{n}^{a}+t_{n}^{b} & =0, \\
g^{a b} & =0 .
\end{aligned}
$$

(3) Slip Mode. Because frictionless contact is considered, the individual tangential traction at the pair of points $a$ and $b$ is still zero, and the sum of normal traction is equal to zero. The gap should be zero:

$$
\begin{aligned}
t_{t}^{a} & =t_{t}^{b}=0, \\
t_{n}^{a}+t_{n}^{b} & =0, \\
g^{a b} & =0 .
\end{aligned}
$$

It should be mentioned that the individual normal contact traction at the pair of points $a$ and $b$ is always less than zero (i.e., compressive). Here, subjected to the local coordinate system of point $b$ on the body $\Omega^{B}$, the normal contact pressure can be expressed as

$$
t_{n}^{a b}=t_{n}^{b}=-t_{n}^{a}
$$

Furthermore, $g^{a b}$ can be expressed as

$$
g^{a b}=g_{0}^{a b}+\left(\mathbf{u}^{a}-\mathbf{u}^{b}\right) \cdot \mathbf{n}^{b},
$$




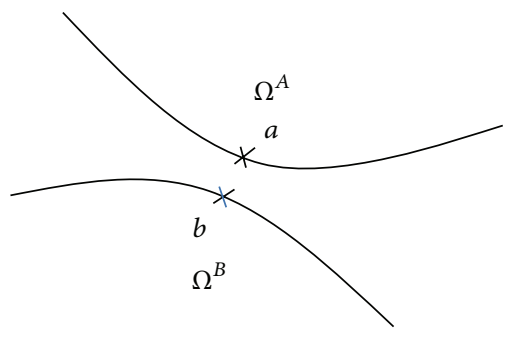

(a) Separation mode

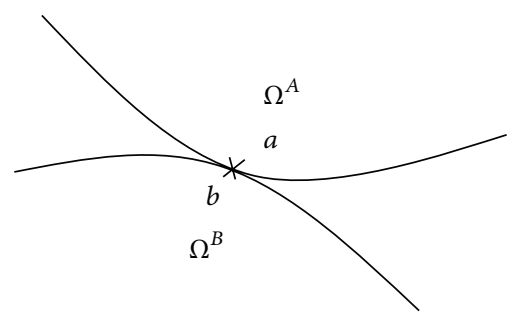

(b) Stick mode

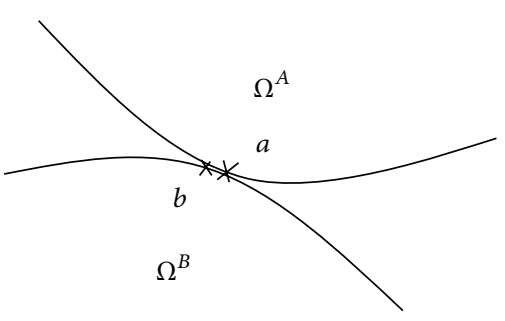

(c) Slip mode

Figure 2: Contact modes.

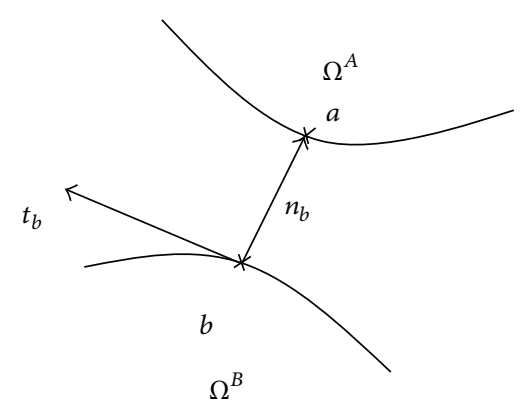

Figure 3: Local coordinate system.

where $g_{0}^{a b}$ defines the initial gap between the points $a$ and $b$, and $\mathbf{u}^{a}$ and $\mathbf{u}^{b}$ define the displacement vector on the bodies of $\Omega^{A}$ and $\Omega^{B}$, respectively. $\mathbf{n}^{b}$ denotes the unit vector at the contacting point of body $\Omega^{B}$.

\subsection{Complementarity Theory for Contact Problems}

2.2.1. Complementarity Problem. The complementary problem [30] is an important optimization problem. It is widely employed in many problems, such as game theory, economy analysis, and traffic equilibrium. It is firstly proposed by Dantzig and Cottle in 1963. It can be stated as follows [31-33].

Definition 1. For a given map $F$ from the $n$-dimensional Euclidean space $\mathbf{R}^{n}$ into itself, find $x \in R^{n}$ satisfying

$$
\begin{aligned}
x & \geq 0, \\
F(x) & \geq 0, \\
x^{T} F(x) & =0 .
\end{aligned}
$$

When $F$ is the form $F(x)=M x+q\left(M \in R^{n \times n}, q \in R^{n}\right)$, the above problem is referred to as the linear complementarity problem (LCP); otherwise, it is called the nonlinear complementarity problem (NCP).

In the past few decades, the complementary problem has attracted much attention because of its wide applications. Consequently, the algorithm has achieved fruitful results and mainly includes the Lemke algorithm [34], homotopy method [35], projection algorithm [36], interior point algorithm [37], and system of equations algorithm [22]. One of the most powerful and popular methods is to reformulate the complementary problem as the system of equations. To construct it, a class of functions, called NCP-functions, defined below, plays an important role.

Definition 2. A function $\phi: R^{2} \rightarrow R$ is called an NCPfunction if it satisfies

$$
\phi(a, b)=0 \Longleftrightarrow a \geq 0, \quad b \geq 0, a b=0 .
$$

Common NCP functions are as follows:

(1) $\varphi(a, b)=\sqrt{a^{2}+b^{2}}-a-b$,

(2) $\varphi(a, b)=-a b+(1 / 2) \min ^{2}\{0, a+b\}$,

(3) $\varphi(a, b)=\min (a, b)$.

The most wonderful feature of NCP function is that it transforms the problem containing two equalities and an inequality into a problem only containing an equality. Therefore, the complex contact problem can be solved by solution of the system of equations.

2.2.2. NCP-Function for Contact Problems. Using $g$ and $t_{n}$ to stand for the gap and contact pressure of any potential contacting pair of points, the normal contact conditions can be described as

$$
\begin{array}{ll}
t_{n}=0 \quad \text { When } g>0, \\
t_{n} \leqslant 0 \quad \text { When } g=0 .
\end{array}
$$

Furthermore, the gap and contact pressure can be described by the following graph, as shown in Figure 4.

According to (8), the following relationships can be achieved:

$$
\begin{aligned}
g & \geq 0, \\
-t_{n} & \geq 0, \\
g \cdot\left(-t_{n}\right) & =0 .
\end{aligned}
$$

Obviously, it is a complementary description between the gap $g$ and minus normal contact stress $-t_{n}$. Consequently, the above description can be expressed by the NCP function mentioned above. Using the function $\varphi(a, b)=-a b+$ $(1 / 2) \min ^{2}\{0, a+b\}$, we can express (9) as

$$
g \cdot\left(-t_{n}\right)+\frac{1}{2} \min ^{2}\left\{0, g-t_{n}\right\}=0 .
$$




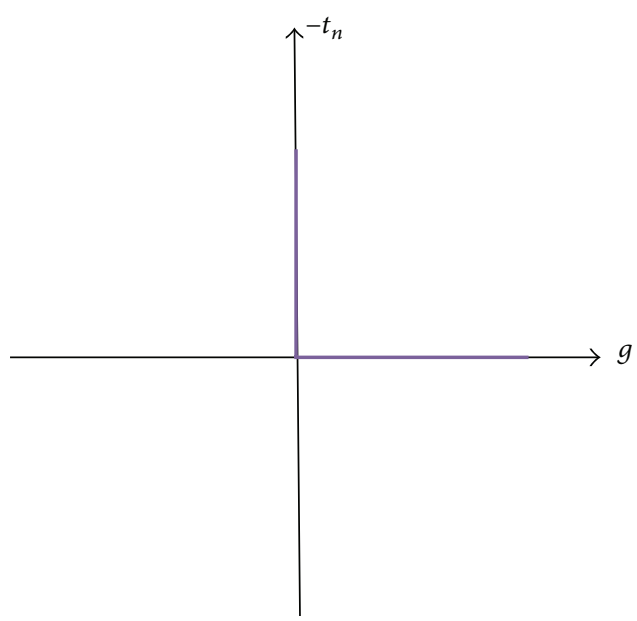

FIGURE 4: Relationship between the gap and normal contact pressure.

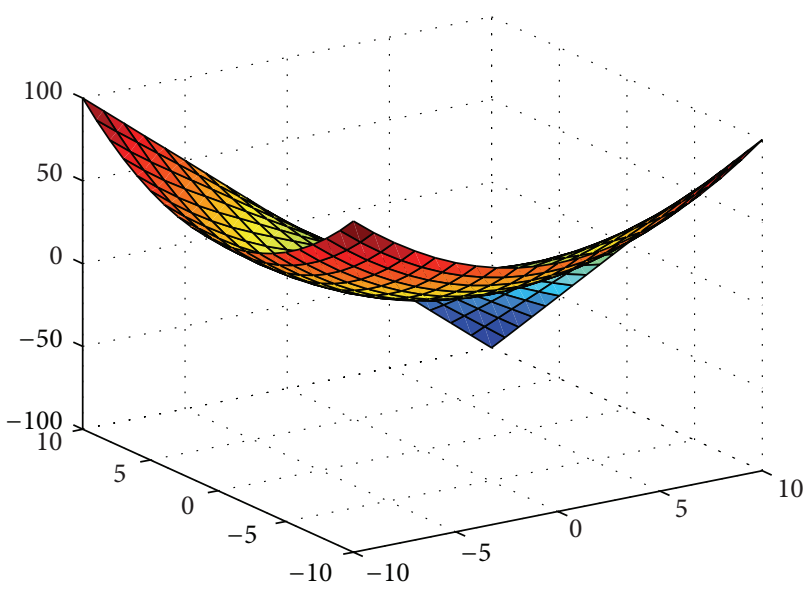

FIgURE 5: The graph of $\varphi(a, b)$ function.

Figure 5 shows the graph of the function above and it can be observed that the proposed function is smooth everywhere and well suitable for contact problems.

\section{Complementarity Problem Formulation by BEM}

The BEM formulation for an elastic continuum is well known and can be found in many classical texts such as Brebbia et al. [38] and Aliabadi [39]. The elastostatic boundary integral equation for a boundary point $p$ with no body force is given as follows:

$$
\begin{gathered}
c_{i j}(P) u_{j}(P)+\int_{\Gamma} p_{i j}(P, Q) u_{j}(Q) d \Gamma(Q) \\
=\int_{\Gamma} u_{i j}(P, Q) P_{j}(Q) d \Gamma(Q),
\end{gathered}
$$

where $P$ is the source point and $Q$ is the field point at the boundary. $c_{i j}$ is the free coefficient of geometry. $u_{i j}(P, Q)$ and $p_{i j}(P, Q)$ represent the fundamental solutions for displacement and traction components, respectively. The boundary $\Gamma$ consists of displacement boundary $\Gamma_{u}$, traction boundary $\Gamma_{t}$, and potential contact region $\Gamma_{c}$.

In order to perform numerical analysis, the boundary is discretized into linear elements. Equation (11) can be conveniently expressed in the following matrix form:

$$
[H]\{\mathbf{u}\}=[G]\{\mathbf{t}\} .
$$

After each domain is treated separately to form the matrix above, the resulting matrices $[H]$ and $[G]$ are coupled together according to the relevant contact conditions. The total matrices for two contact bodies can be written as follows:

$$
\left[\begin{array}{cc}
{[H]^{A}} & 0 \\
0 & {[H]^{B}}
\end{array}\right]\left[\begin{array}{c}
{[\mathbf{u}]^{A}} \\
{[\mathbf{u}]^{B}}
\end{array}\right]=\left[\begin{array}{cc}
{[G]^{A}} & 0 \\
0 & {[G]^{B}}
\end{array}\right]\left[\begin{array}{l}
{[\mathbf{t}]^{A}} \\
{[\mathbf{t}]^{B}}
\end{array}\right],
$$

where the superscripts $A$ and $B$ refer to the two bodies in contact. Note that under this arrangement the matrices are not fully populated. After the application of boundary conditions, (13) can be recast as

$$
[A]\{x\}=\{f\},
$$

where $\{x\}$ denotes the boundary unknowns and $\{f\}$ is the contribution of known boundary variables, that is, values prescribed by the boundary conditions. It is noted that the matrix $[A]$ is not a square matrix, so the contact conditions will be incorporated.

For any node pair, there are four unknowns for each node, namely, normal and tangential displacements and tractions, $\left\{u_{t}, u_{n}, t_{t}, t_{n}\right\}$, and they are referred to a local coordinate system. Except for the two boundary integral equations for every contact node, other four complementary equations will be listed to solve the system of equations.

Take the pair of nodes $a$ and $b$ as an example; the following relationships hold:

$$
\begin{aligned}
t_{t}^{a} & =0, \\
t_{t}^{b} & =0, \\
t_{n}^{a}+t_{n}^{b} & =0, \\
g \cdot\left(-t_{n}\right)+\frac{1}{2} \min ^{2}\left\{0, g-t_{n}\right\} & =0 .
\end{aligned}
$$

So far, the numbers of unknowns are equal to the number of equations, and the system of equations can be solved by Newton method [40].

Consequently, the calculation process has been exhibited as shown in Figure 6, and the corresponding compute program by BEM is formulated. It should be noted that the proposed algorithm does not need to judge the contact modes in the calculating process. After solving the system of equations, the displacements and tractions for any potential node pair are shown, and the contact mode is clear at a glance. 


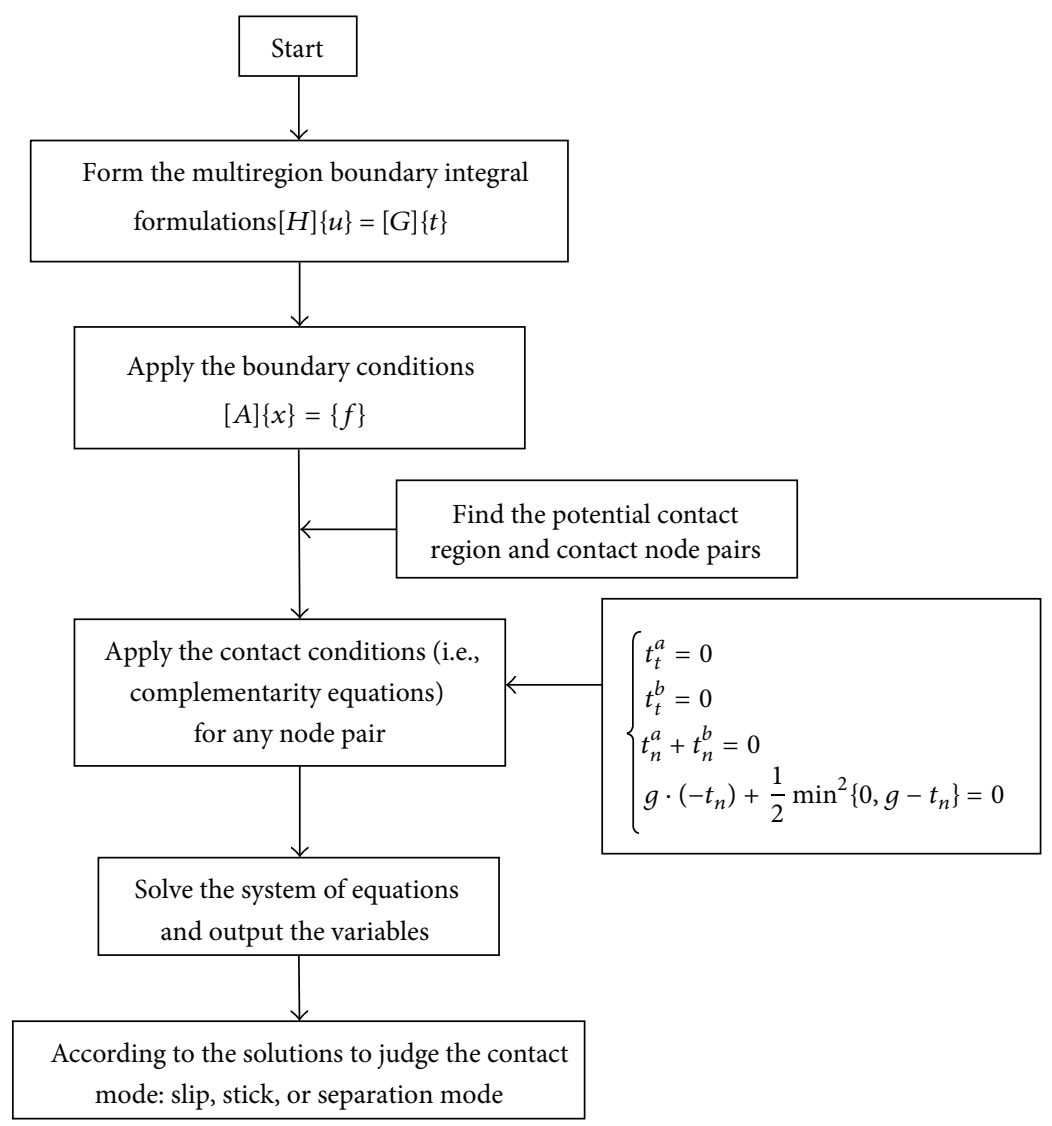

FIGURE 6: Flow chart of the calculation process.

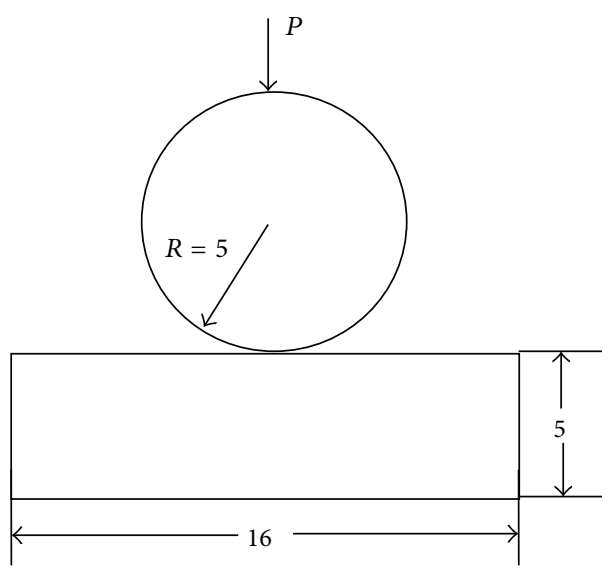

(a) Geometry

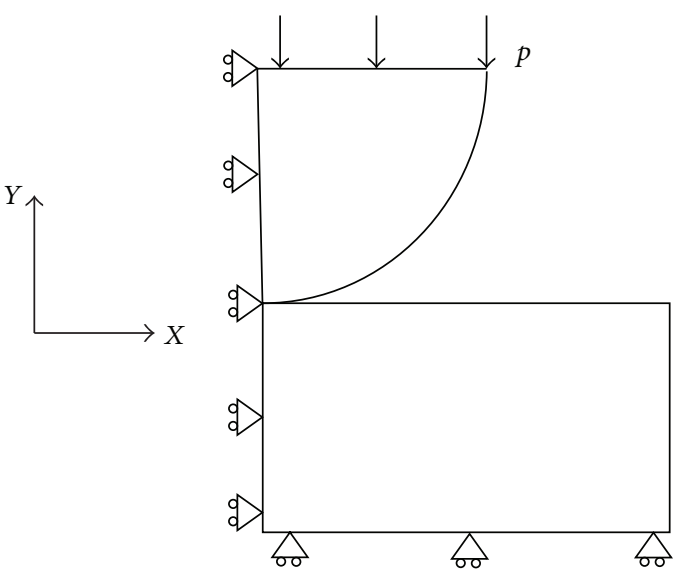

(b) Simplified model

Figure 7: Contact of cylinder and base (unit/m).

\section{Numerical Examples}

It should be mentioned that contact problem widely exists in the static and dynamic problem. The difference between static and dynamic problem is that whether the effect of acceleration can be ignored or not. Although this paper mainly considers the static contact problem, the proposed method can be easily extended to dynamic problem.
A computer program by BEM is applied to two practical contact problems. The BEM results are compared to analytical solutions and FEM results to establish their accuracy.

Example 1 (cylinder and base contact problem). In this problem, a cylinder with a radius of $5 \mathrm{~m}$ is pressed against a $5 \mathrm{~m}$ deep base under plane strain conditions shown in Figure $7(\mathrm{a})$. Due to the symmetry of problem, only the 


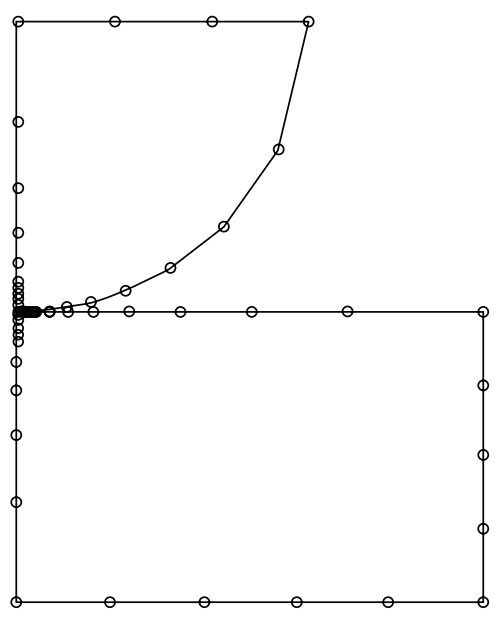

(a) Boundary discretization

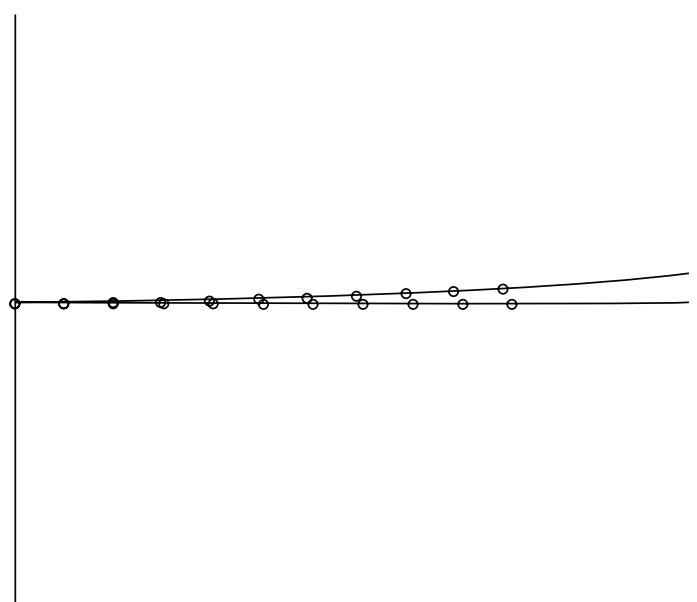

(b) Fine discretization of the potential contact region

FIgURE 8: Boundary element discretization.

quarter of cylinder and the half of base have been modeled as shown in Figure $7(\mathrm{~b})$. Here, the uniform load along the cylinder's top per unit axial length $(P=100 \mathrm{KN} / \mathrm{M})$ can be expressed by the load $P$ divided by the diameter of cylinder $\left(p=100 /(2 * 5)=10 \mathrm{KN} / \mathrm{M}^{2}\right)$. Both the cylinder and base are given the same properties: elastic modulus $E=30 \mathrm{MPa}$ and Poisson's ratio $v=0.3$.

Such problems are referred to as Hertz-type or Hertzian contact problems, and the analytical solution can be given as follows [41]:

$$
\begin{aligned}
a & =\sqrt{\frac{4 p \bar{R}}{\pi E^{*}}} \\
p(x) & =p_{\max }\left(1-\frac{x^{2}}{a^{2}}\right)^{1 / 2}, \quad-a \leq x \leq a, \\
p_{\max } & =\frac{\pi}{4} \sqrt{\frac{p E^{*}}{\pi \bar{R}}},
\end{aligned}
$$

where $a$ is the half-width of the contact, $p_{\max }$ is the maximum pressure at the center of the contact, and $p(x)$ is the pressure distribution of the contact. $E^{*}$ and $\bar{R}$ are given by

$$
\begin{aligned}
\frac{1}{E^{*}} & =\frac{1-v_{1}^{2}}{E_{1}}+\frac{1-v_{2}^{2}}{E_{2}}, \\
\frac{1}{\bar{R}} & =\frac{1}{R_{1}}+\frac{1}{R_{2}} .
\end{aligned}
$$

In the boundary element analysis, the cylinder and base consist of 31 and 37 linear elements as shown in Figure 8(a), respectively. In order to obtain more accurate stress, a very fine mesh of the potential contact region is designed in Figure $8(\mathrm{~b})$. The boundary conditions are as follows: the vertical displacements are fixed at the bottom of the base, and

\begin{tabular}{|c|c|c|}
\hline $\begin{array}{l}\text { Element size in potential } \\
\text { contact region } / \mathrm{m}\end{array}$ & Contact length $/ \mathrm{m}$ & $\mathrm{Gap} / \mathrm{mm}$ \\
\hline \multirow{11}{*}{0.03} & 0 & 0 \\
\hline & 0.03 & 0 \\
\hline & 0.06 & 0 \\
\hline & 0.09 & 0 \\
\hline & 0.12 & 0 \\
\hline & 0.15 & 0 \\
\hline & 0.18 & 0 \\
\hline & 0.21 & 0.09 \\
\hline & 0.24 & 0.65 \\
\hline & 0.27 & 1.55 \\
\hline & 0.3 & 2.72 \\
\hline \multirow{7}{*}{0.05} & 0 & 0 \\
\hline & 0.05 & 0 \\
\hline & 0.1 & 0 \\
\hline & 0.15 & 0 \\
\hline & 0.2 & 0 \\
\hline & 0.25 & 0.96 \\
\hline & 0.3 & 2.76 \\
\hline \multirow{4}{*}{0.1} & 0 & 0 \\
\hline & 0.1 & 0 \\
\hline & 0.2 & 0 \\
\hline & 0.3 & 2.81 \\
\hline
\end{tabular}
the horizontal displacements are fixed for the left boundaries of the cylinder and base.
TABLE 1: Gap between node pairs in potential contact region for three kinds of element size.

To compare the effects of discretization in potential contact region, three kinds of fine element size in potential contact region have been given, that is, element size $=0.03 \mathrm{~m}$, $0.05 \mathrm{~m}$, and $0.1 \mathrm{~m}$. Consequently, the results of proposed method have been listed in Table 1. It is shown that the half-width of contact length is about $0.2 \mathrm{~m}$, and the gap is 


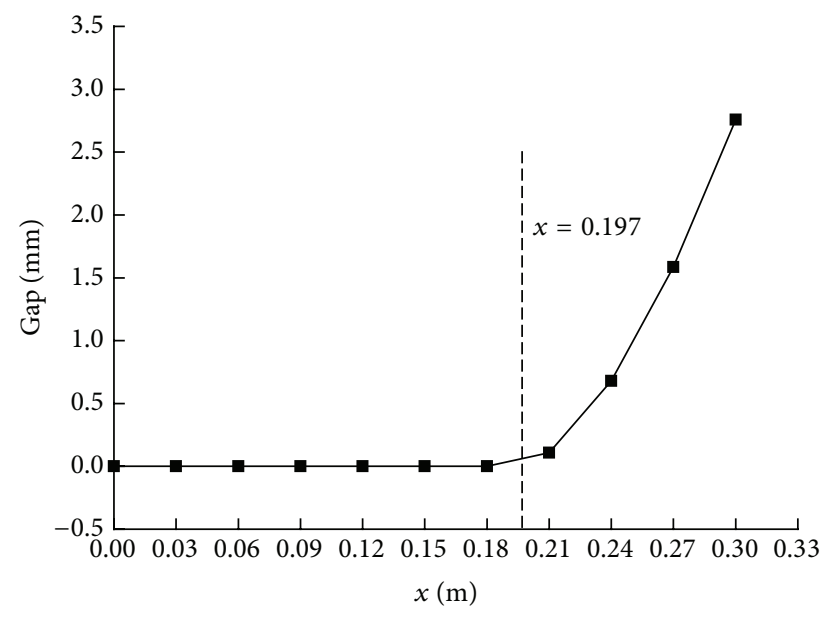

FIGURE 9: Gap between nodes in the potential contact region.

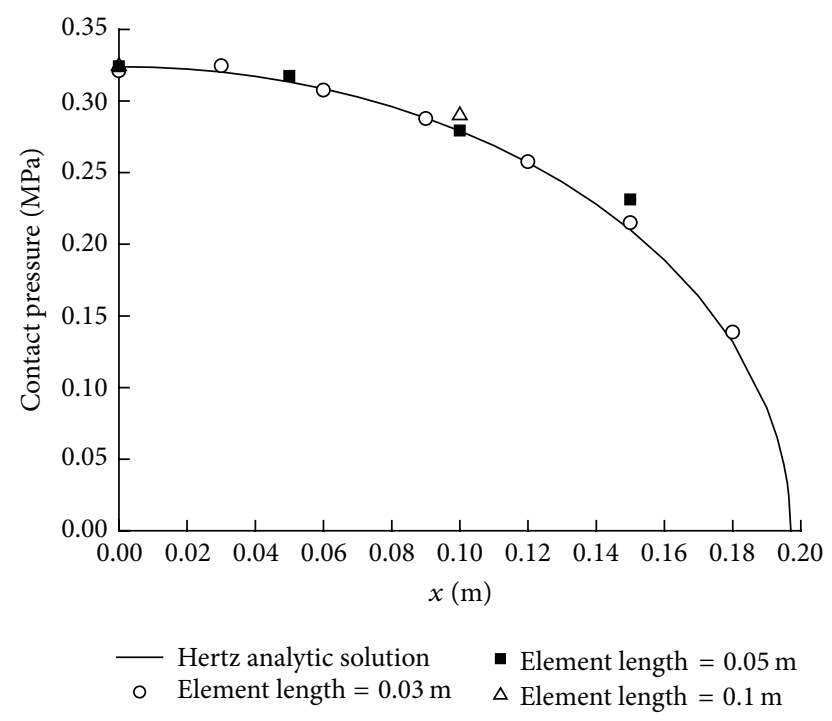

Figure 10: Contact pressure versus contact length.

not sensitive in element size. Because the analytic solutions exist, the result of proposed method for element size $0.03 \mathrm{~m}$ has been compared with them in Figure 9. According to the formula, the half-width of the contact $a$ is equal to $0.197 \mathrm{~m}$.

Figure 10 plots the contact pressure distributions along the contact length, and clearly there is excellent agreement between the analytical solution and the proposed method. It is also found that when the element size in potential contact region is smaller, the result for contact pressure is more accurate.

In general, the proposed method can give the satisfactory results. To obtain accurate results, the small element size in potential contact region is recommended.

Example 2 (laminated beam problem). Considering a laminated beam problem [42] as shown in Figure 11, the size of every beam is $10 \times 1 \times 1 \mathrm{~m}$. The parameters of material are as follows: Young's modulus $E=1500 \mathrm{MPa}$ and Poisson's ratio $v=0.25$. There are two cases to consider. In case 1 , the point $A$

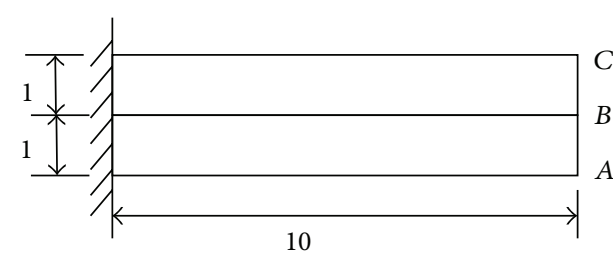

Figure 11: A cantilever with beam-over-beam.

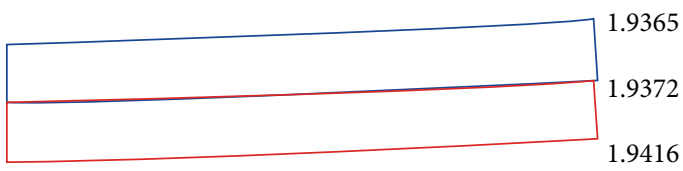

(a) Downward load

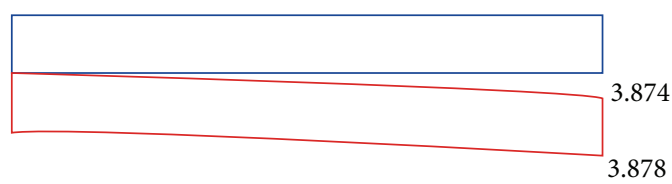

(b) Upward load

FIGURE 12: Deformation pictures (unit/mm).

TABLE 2: Displacements under different load (unit/mm).

\begin{tabular}{lccc}
\hline Points & $A$ & $B$ & $C$ \\
\hline Upward & & & \\
$\quad$ Proposed method & 1.942 & 1.937 & 1.937 \\
$\quad$ Reference [29] & 2.011 & 2.007 & 2.006 \\
$\quad$ Reference [30] & 1.989 & 1.983 & 1.982 \\
Downward & & & \\
$\quad$ Proposed method & 3.878 & 3.874 & 0 \\
$\quad$ Reference [29] & 4.016 & 4.013 & 0 \\
$\quad$ Reference [30] & 3.969 & 3.964 & 0 \\
\hline
\end{tabular}

has a downward concentrate force $P=1.5 \mathrm{KN}$, while in case 2 the force is upward. The problem is considered under plane stress condition.

In order to simulate the concentrate load $P$, a distributed load is applied vertically along a very small element near the point $A$. To investigate the accuracy, the displacements of points $A, B$, and $C$ have been selected. Figure 12 shows the deformation under different load, and the results have satisfactory agreement with the results by Zheng et al. [42] and $\mathrm{Li}$ [43]. Table 2 lists displacements of specified three points.

\section{Conclusions}

Frictionless contact problems in two-dimensional space are formulated by complementarity theory, where the system of equations is established by the nonlinear complementary functions and boundary integral equations. This algorithm by BEM is established. The accuracy and effectiveness of the method have been demonstrated by two numerical examples, and the effect of discretization has also been studied in 
the Hertzian contact problem. The results show that this technique is very competitive and elegant.

Several extensions of the current work are possible. The presented method can be easily extended to contact problems with friction or involving inelastic materials.

\section{Conflict of Interests}

The authors declare that there is no conflict of interests regarding the publication of this paper.

\section{Acknowledgments}

The authors gratefully acknowledge the financial support of Natural Science Foundation of China (no. 41202226) and National 973 Project of China (no. 2011CB013505).

\section{References}

[1] P. Wriggers, Computational Contact Mechanics, Springer, Berlin, Germany, 2006.

[2] N. Kikuchi and J. T. Oden, Contact Problems in Elasticity: A Study of Variational Inequalities and Finite Element Method, Society for Industrial Mathematics, Philadelphia, Pa, USA, 1987.

[3] T. F. Conry and A. Seireg, "A mathematical programming method for the design of elastic bodies in contact," Journal of Applied Mechanics, vol. 93, no. 1, pp. 387-392, 1971.

[4] N. Chandrasekaran, W. E. Haisler, and R. E. Goforth, "Finite element analysis of Hertz contact problem with friction," Finite Elements in Analysis and Design, vol. 3, no. 1, pp. 39-56, 1987.

[5] K.-J. Bathe and A. Chaudhary, "A solution method for planar and axisymmetric contact problems," International Journal for Numerical Methods in Engineering, vol. 21, no. 1, pp. 65-88, 1985.

[6] C. M. Zhu, "A finite element-mathematical programming method for elastoplastic contact problems with friction," Finite Elements in Analysis and Design, vol. 20, no. 4, pp. 273-282, 1995.

[7] Y. S. Jiang and C. Su, "Numerical analysis methods for solving contact problems in engineering," Water Power, vol. 36, no. 4, pp. 75-78, 2010.

[8] M. U. Rahman, R. E. Rowlands, R. D. Cook, and T. L. Wilkinson, "An iterative procedure for finite-element stress analysis of frictional contact problems," Computers and Structures, vol. 18, no. 6, pp. 947-954, 1984.

[9] A. Francavilla and O. C. Zienkiewicz, "A note on numerical computation of elastic contact problems," International Journal for Numerical Methods in Engineering, vol. 9, no. 4, pp. 913-924, 1975.

[10] H. Gun and X.-W. Gao, "Analysis of frictional contact problems for functionally graded materials using BEM," Engineering Analysis with Boundary Elements, vol. 38, pp. 1-7, 2014.

[11] H. Gun, "Isotropic damage analysis of frictional contact problems using quadratic meshless boundary element method," International Journal of Mechanical Sciences, vol. 80, pp. 102108, 2014.

[12] K. Yamazaki, J. Sakamoto, and S. Takumi, "Penalty method for three-dimensional elastic contact problems by boundary element method," Computers and Structures, vol. 52, no. 5, pp. 895-903, 1994.

[13] P. Papadopoulos and J. M. Solberg, "A Lagrange multiplier method for the finite element solution of frictionless contact problems," Mathematical and Computer Modelling, vol. 28, no. 4-8, pp. 373-384, 1998.

[14] J. C. Simo and T. A. Laursen, "An augmented lagrangian treatment of contact problems involving friction," Computers \& Structures, vol. 42, no. 1, pp. 97-116, 1992.

[15] X. W. Du, Augmented Lagrangian function methods for solving constrained optimization problems [Ph.D. thesis], Shanghai University, Shanghai, China, 2007.

[16] J.-S. Pang, "A B-differentiable equation-based, globally and locally quadratically convergent algorithm for nonlinear programs, complementarity and variational inequality problems," Mathematical Programming, vol. 51, no. 1-3, pp. 101-131, 1991.

[17] Y. Kanno and M. Ohsaki, "A non-interior implicit smoothing approach to complementarity problems for frictionless contacts," Computer Methods in Applied Mechanics and Engineering, vol. 200, no. 9-12, pp. 1176-1185, 2011.

[18] S. Šimunovic and S. Saigal, "Frictionless contact with BEM using quadratic programming," Journal of Engineering Mechanics, vol. 118, no. 9, pp. 1876-1891, 1992.

[19] S. Šimunovic and S. Saigal, "Frictional contact formulation using quadratic programming," Computational Mechanics, vol. 15, no. 2, pp. 173-187, 1994.

[20] X.-A. Kong, A. Gakwaya, A. Cardou, and L. Cloutier, "A numerical solution of general frictional contact problems by the direct boundary element and mathematical programming approach," Computers \& Structures, vol. 45, no. 1, pp. 95-112, 1992.

[21] S. De-Song, S. Huan-Chun, and X. Shou-Ze, "Formulation of boundary element-linear complementary equation for the frictional elastic contact problems," Applied Mathematics and Mechanics, vol. 11, no. 12, pp. 1105-1112, 1990.

[22] O. L. Mangasarian, "Equivalence of the complementarity problem to a system of nonlinear equations," SIAM Journal on Applied Mathematics, vol. 31, no. 1, pp. 89-92, 1976.

[23] C. A. Brebbia and J. Dominguez, Boundary Elements: An Introductory Course, Computational Mechanics Publications, McGraw-Hill, Southampton, NY, USA, 1992.

[24] D. Martín and M. H. Aliabadi, "Boundary element analysis of two-dimensional elastoplastic contact problems," Engineering Analysis with Boundary Elements, vol. 21, no. 4, pp. 349-360, 1998.

[25] T. Andersson, "The boundary element method applied to two-dimensional contact problems with friction," in Boundary Element Methods, vol. 3 of Boundary Elements, pp. 239-258, Springer, Berlin, Germany, 1981.

[26] T. Andersson, B. Fredriksson, and B. G. Allan-Persson, "The boundary element method applied to two-dimensional contact problems," in New Developments in Boundary Element Methods, pp. 239-258, CML Publications, Southampton, UK, 1980.

[27] T. Andersson and B. G. Allan-Persson, "The boundary element method applied to two-dimensional contact problems," Progress in Boundary Element Methods, vol. 2, pp. 136-157, 1983.

[28] Y.-G. Li, Q.-X. Huang, G.-X. Shen, H. Xiao, S.-Q. Pang, and J.-M. Wang, "Simulation of strip rolling using elastoplastic contact BEM with friction," Journal of Iron and Steel Research, International, vol. 15, no. 1, pp. 34-38, 2008.

[29] B. Y. Keum and Y. J. Liu, "Analysis of 3-D frictional contact mechanics problems by a boundary element method," Tsinghua Science and Technology, vol. 10, no. 1, pp. 16-29, 2005.

[30] F. Facchinei and J. S. Pang, Finite-Dimensional Variational Inequalities and Complementarity Problems, Springer, New York, NY, USA, 2003. 
[31] O. L. Mangasarian, "Solution of symmetric linear complementarity problems by iterative methods," Journal of Optimization Theory and Applications, vol. 22, no. 4, pp. 465-485, 1977.

[32] S. Karamardian, "Generalized complementarity problem," Journal of Optimization Theory and Applications, vol. 8, pp. 161-168, 1971.

[33] P. T. Harker and J.-S. Pang, "Finite-dimensional variational inequality and nonlinear complementarity problems: a survey of theory, algorithms and applications," Mathematical Programming, vol. 48, no. 2, pp. 161-220, 1990.

[34] C. E. Lemke and J. T. Howson, "Equilibrium points of bimatrix games," SIAM Journal on Applied Mathematics, vol. 12, pp. 413423, 1964.

[35] L. T. Watson, "Solving the nonlinear complementarity problem by a homotopy method," SIAM Journal on Control and Optimization, vol. 17, no. 1, pp. 36-46, 1979.

[36] Y. C. Cheng, "On the gradient-projection method for solving the nonsymmetric linear complementarity problem," Journal of Optimization Theory and Applications, vol. 43, no. 4, pp. 527541, 1984.

[37] M. Kojima, N. Megiddo, and Y. Ye, "An interior point potential reduction algorithm for the linear complementarity problem," Mathematical Programming, vol. 54, no. 1-3, pp. 267-279, 1992.

[38] C. A. Brebbia, J. C. F. Telles, and L. C. Wrobel, Boundary Element Techniques, Springer, Berlin, Germany, 1984.

[39] M. H. Aliabadi, The Boundary Element Method: Applications in Solids and Structures, John Wiley \& Sons, Hoboken, NJ, USA, 2002.

[40] Q. Y. Li, N. C. Wang, and D. Y. Yi, Numerical Analysis, Tsinghua Press, Beijing, China, 2008.

[41] G. Karami, A Boundary Element Method for Two-Dimensional Contact Problems, Lecture Notes in Engineering, Springer, Berlin, Germany, 1987.

[42] H. Zheng, C.-F. Lee, X. R. Ge, and Z. Yue, "A mixed finite element solution for interface problems," Chinese Journal of Rock Mechanics and Engineering, vol. 21, no. 1, pp. 1-8, 2002.

[43] C. G. Li, Strength of brittle rock and simulation of structural surfaces [Ph.D. thesis], Institute of Rock and Soil Mechanics, Chinese Academy of Sciences, Beijing, China, 2005. 


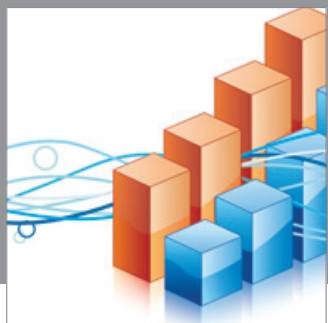

Advances in

Operations Research

mansans

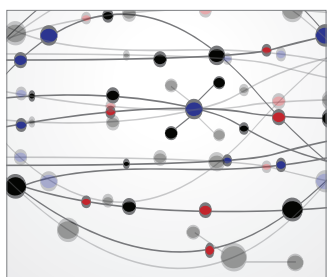

The Scientific World Journal
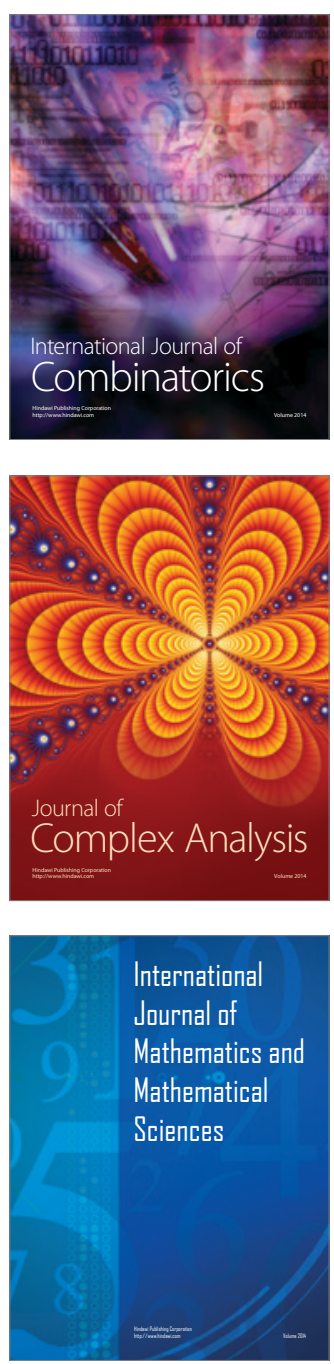
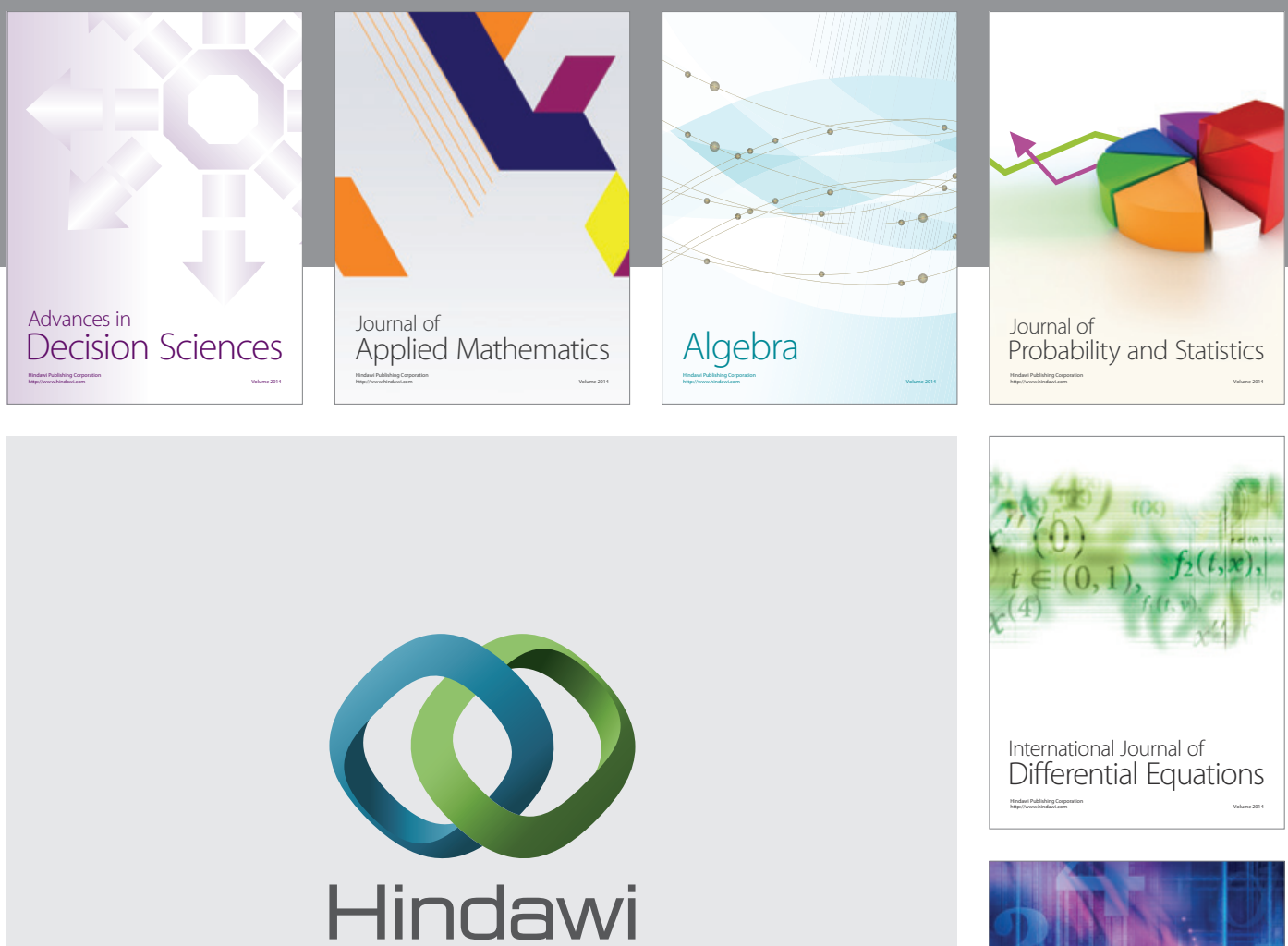

Submit your manuscripts at http://www.hindawi.com
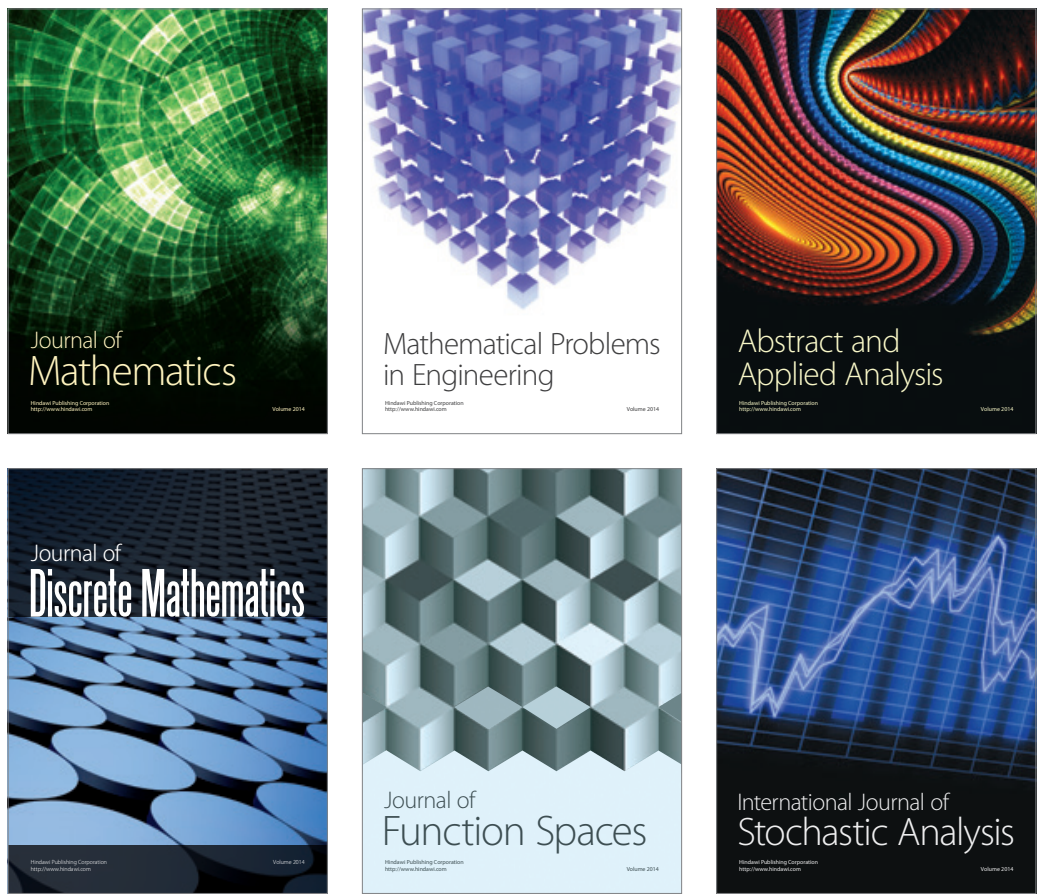

Journal of

Function Spaces

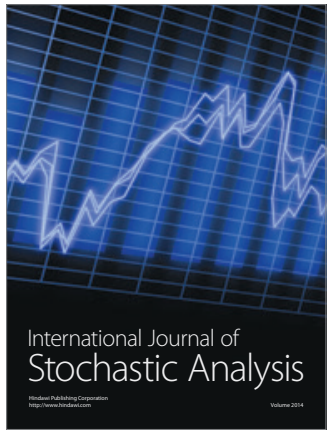

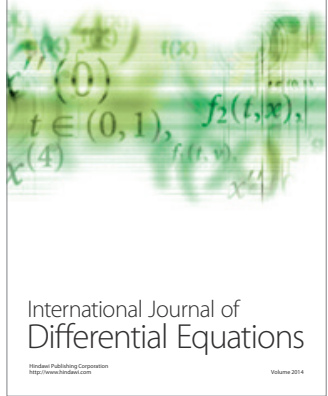
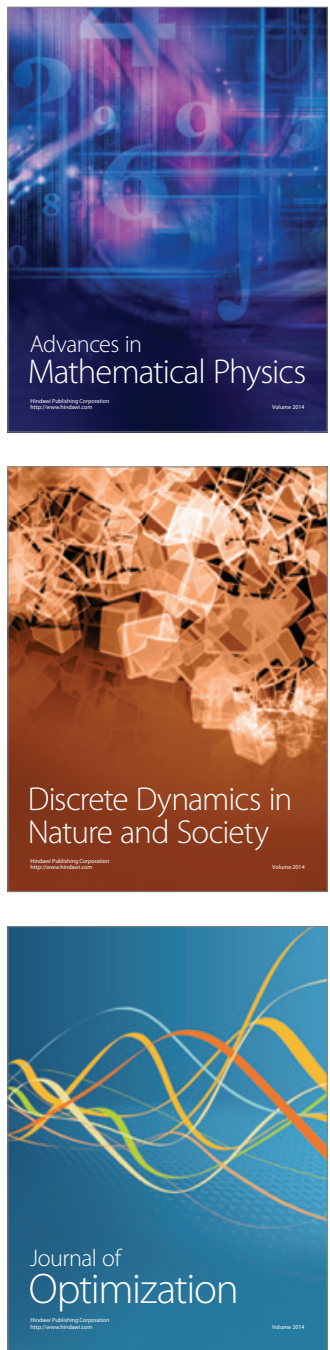